\title{
A STUDY OF THE DECAY PHASE OF AN X-RAY FLARE ON ALGOL
}

\author{
R. Mewe, ${ }^{1}$ G.II.J. van den Oord, ${ }^{2}$ J. Jakimiec ${ }^{3}$ \\ ${ }^{1}$ Laboratory for Space Rescarch, Utrecht, The Netherlands \\ ${ }^{2}$ Dept. of Physics and Astronomy, University of Gliasgow, U.K., Scotland \\ ${ }^{3}$ Astronomical Institute, Wroclaw University, Poland
}

\begin{abstract}
We have re-analyzed the X-ray flare on Algol which was observed with EXOSAT (White et al. (1986)). The common practice of estimating loop volume and length from the decay time of the flare is discussed extensively. We show that during the decay phase of the flare both scaling laws for coronal loops are valid. This implies a unique determination of loop volume and length and allows a check whether additional heating occurs in the decay phase of a flare.
\end{abstract}

\section{Determination of loop volume from cooling time scales}

A serious limitation for the interpretation of stellar flares is the unknown volume. Normally this is obtained by equating the decay time of the light curve to the conductive or radiative loss time, This practice is wrong because the light curve decay time is not equal to the characteristic time on which the thermal energy decreases.

Assuming that the flare occurs in $N$ identical loops with total length $L$, height $I I=L / \pi$ and diameter $d$ to length $L$ ratic, $\alpha=d / L$, thre flare volume $V$ is given by

$$
V=\frac{1}{4} \pi^{4} H^{3} N \alpha^{2} \quad \mathrm{~cm}^{3} .
$$

The energy equation during flare decay is given by

$$
\frac{d\left(3 n_{c} k T\right)}{d t}=-E_{r}-E_{c} \quad \operatorname{erg} \mathrm{cm}^{-3} \mathrm{~s}^{-1}
$$

with the radiative (Mewe et al. 1985, 1986; $T>20 \mathrm{MK}$ ) and conductive losses (Dowdy et al. 1985):

$$
E_{r}=n_{e}^{2} \Psi_{0} T^{-\gamma}=1.8610^{-25} n_{e}^{2} T^{0.25}, \quad E_{c}=\frac{16}{7} \kappa_{0} \frac{T^{7 / 2}}{L^{2}}=2.010^{-6} \frac{T^{7 / 2}}{L^{2}},
$$

where temperature $T$ in $\mathrm{K}$, loop length $L$ in $\mathrm{cm}$ and electron density $n_{e}$ in $\mathrm{cm}^{-3}$.

Define the radiative and conductive loss times by

$$
\tau_{r}=\frac{3 n_{e} k T}{E_{r}}, \quad \tau_{c}=\frac{3 n_{e} k T}{E_{c}}
$$

The observed quantities are the decay time $\tau_{d}$ of the X-ray light curve and the decay time $\tau_{T}$ of the temperature, i.e.

$$
E_{r}(t)=E_{r 0} e^{-t / \tau_{d}}, \quad T(t)=T_{0} e^{-t / \tau_{\tau}},
$$

with index 0 indicating the start of the decay phase at tirne $t=0$. Using Eqs. (1), (1) and (5) we can evaluate Eq. (2) at $t=0$, resulting in (see Van den Oord et al. 1988)

$$
\frac{1}{\tau_{e f \rho}}=\frac{7 / 8}{\tau_{T}}+\frac{1 / 2}{\tau_{d}}=\frac{1}{\tau_{r}}+\frac{1}{\tau_{c}}
$$

or

$$
H^{3 / 2}-A H-B=0
$$


with

$$
A=\frac{8}{21} \frac{\kappa_{0}}{k} \frac{T_{0}^{5 / 2}}{E M_{0}^{1 / 2}} \tau_{e \jmath \rho} N^{1 / 2} \alpha, \quad B=\frac{2}{3 \pi^{2}} \frac{\Psi_{0}}{k} \frac{E M^{1 / 2}}{T^{0.75}} \frac{\tau_{e \rho}}{N^{1 / 2} \alpha},
$$

where $E M=\int u_{e}^{2} d V$ is the cmission measure. In Fig. 1 we plot the loop height $I$ as derived from observations (Van den Oord and Mewe 1988) against $N \alpha_{0.1}^{2}$ ("cooling curve"). The loop height and the volume $V$ still depend on the assumed $N \alpha^{2}$, which reflects the unknown ratio $\left(\mu^{\prime}=\tau_{r} / \tau_{c}\right)$ of radiation and conduction cooling times. Since $\mu^{\prime} \propto H N \alpha^{2}$, determining $\mu^{\prime}$ implies determining the flare geometry.

\section{Cooling of flare loop according to scaling laws}

The situation changes when the loop cools quasi-statically according to the scaling laws. For a loop heated at constant rate $E_{11}$ the local energy balance is:

$$
0=E_{H}-E_{\mathrm{r}}-E_{c} \quad \operatorname{erg} \mathrm{cm}^{-3} \mathrm{~s}^{-1} .
$$

For a uniform pressure $p$ in the loop, eq. (7) results in two scaling laws (see e.g. Kuin and Martens 1982):

$$
E_{H}=n^{2} \Psi_{0} I^{0.25}(\mathrm{SL} 1), \quad n\left(\frac{L}{2}\right)=\mu^{-1 / 2}\left(\frac{\kappa_{0}}{\Psi_{0}}\right)^{1 / 2} T^{13 / 8} \text { (SL2). }
$$

The first scaling law expresses that the average heating in the loop is balanced by the average radiative losses, whereas the second scaling law gives a fired ratio $\mu=(7 / 4) \mu^{\prime}=(7 / 4) \tau_{r} / \tau_{c}$, which depends only on the exponent of the radiation loss function. In our case: $\mu=0.18$ (or $\mu^{\prime}=0.1$ ), which is confirmed by the observations of the decay of the X-ray flare on Algol (Van den Oord and Mewe 1988). In Fig. 1 the curve $\mu^{\prime}=0.1$ is drawn and for comparison also curves $\mu^{\prime}=1$ (this is often assumed, but not correct), and $\mu^{\prime}=10$. The temperature $T$ and electron density $n$ appearing in the scaling laws are the values at the inflection point of the temperature distribution, where the radiative losses equal the mean over the loop. Therefore these values are the mean values for the loop that can be identified with the corresponding observed quantities. The local energy balance for a loop in the decay phase of a flare reads

$$
\frac{d\left(\frac{3}{2} p\right)}{d t}=\varepsilon_{H}-E_{r}-E_{c},
$$

with $\varepsilon_{I I}$ accounting for a uniform additional heating in the decay phase. Comparing Eqs. (7) and (8) gives

$$
E_{H}=-\frac{d\left(\frac{3}{2} p\right)}{d t}+\varepsilon_{H}
$$

where $E_{I I}$ is now a slowly varying function of time. From Eqs. (8) and (10) we find that the time evolution of a loop cooling quasi-statically is given by

$$
\begin{aligned}
& \frac{d T(t)}{d t}=\frac{4}{63} \frac{L}{k}\left(\frac{\mu \Psi_{0}}{\kappa_{0}}\right)^{1 / 2} T(t)^{-13 / 8}\left[\varepsilon_{H}-\frac{4 \kappa_{0}}{L^{2} \mu} T(t)^{7 / 2}\right] \\
& n(t)(L / 2)=\left(\frac{\kappa_{0}}{\mu \Psi_{0}}\right)^{1 / 2} T(t)^{13 / 8}
\end{aligned}
$$

The solution of Eq. (11a) is

$$
\sum_{k=0}^{\infty} \frac{1}{4 k+1}\left(\frac{\varepsilon_{H}}{n_{0}^{2} \Psi_{0} T_{0}^{0.25}}\right)^{k}\left[\left(\frac{T_{0}}{T(t)}\right)^{4 k+1}-1\right]=\frac{1}{3} \frac{t}{\tau}
$$

witl

$$
\tau=\frac{3 k L}{2}\left(\frac{\mu}{\Psi_{0} \kappa_{0}}\right)^{1 / 2} T_{0}^{-7 / 8} \mathrm{~s}
$$




\section{Rosults}

By fitting solution (12) to the observations we found that $\tau=5618 \mathrm{~s}$ and $\varepsilon_{J I}=0$ (i.e. no additional heating), resulting in $V=1.410^{31} \mathrm{~cm}^{3}, L=1.610^{11} \mathrm{~cm}$ and $N \alpha^{2}=0.004$ (cf. also Fig. $1, \mu^{\prime}=0.1$ ). The resulting fits to the observed values of temperature $T(t)$ and emission measure $E M(t)$ are shown in Fig. 2. We note that our method contains no free parameters and therefore results in a unique determination of the flare parameters. Eqs. (11) allow us to express the evolution of all relevant physical quantities in an analytic form (see table 1 , which gives all relevant flare parameters $T, n$, velocity $v$, mass flux and energies).

We successfully applied the model for two-ribbon flares by Kopp and Poletto (1984) to the initial phase of the flare. Details about the application of this model and about the quasi-static cooling treatment can be found in Van den Oord and Mewe (1988).

\section{References}

Dowdy Jr., J.F., Moore, R.I., Wu, S.T.: 1985, Solar Phys. 99, 79

Kopp, R.A., Poletto, G.: 1984, Solar Phys. 93, 351

Kuin, N.P.M., Martens, P.C.II.: 1982, Astron. Astrophys. 108, L1

Mewe, R., Gronenschild, E.II.B.M., van den Oord, G.II.J.: 1985, Astron. Astrophys. Ser. 62, 197

Mewe, R., Lemen, J.R., van den Oord, G.II.J.: 1986, Astron. Astrophys. Suppl. Ser. 65, 511

Van den Oord, G.JI.J., Mewe, R.: 1988, Astron. Astrophys., in press.

Van den Oord, G.II.J., Mewe, R., Brinkmar, A.C.: 1988, Astron. Astrophys., in press.

White, N.E., Culhane, J.L., Parmar, A.N., Kellett, Kahn, S., van den Oord, G.H.J., Kuijpers, J.: 1986, Astrophys. J. 301, 262

Table 1. Parameters of the decay phase of the X-ray flare on Algol

\begin{tabular}{|c|c|c|c|}
\hline \multirow[b]{2}{*}{ temperature } & \multirow[b]{2}{*}{$T=T_{0}\left\{1+\frac{1}{3 \tau}\right\}^{-\theta / 7}$} & \multicolumn{2}{|c|}{$t=0^{a l}$} \\
\hline & & 57.6 & MK \\
\hline density & $n=n_{0}\left\{1+\frac{1}{3 \tau}\right\}^{-13 / 7}$ & $2.6210^{11}$ & $\mathrm{~cm}^{-3}$ \\
\hline velocity $(b)$ & $v=-\frac{L d n}{2 n d t}=\frac{13}{21} v_{0}\left(1+\frac{t}{3 \tau}\right\}^{-1}$ & $8810^{5}$ & $\mathrm{~cm} \mathrm{~s}^{-1}$ \\
\hline mass flux & $n v=\frac{13}{21} n_{0} v_{0}\left\{1+\frac{t}{3 r}\right\}^{-20 / 1}$ & $2.310^{18}$ & $\mathrm{~cm}^{-2} \mathrm{~s}^{-1}$ \\
\hline kinetic energy & $\frac{1}{2} \rho v^{2}=\frac{1}{2} m_{H} n_{0} v_{0}^{2}\left\{1+\frac{t}{3 \tau}\right\}^{-27 / 7}$ & 16.98 & $\mathrm{erg} \mathrm{cm}^{-3}$ \\
\hline thermal energy & $\frac{3}{2} p=3 n_{0} k T_{0}\left[1+\frac{1}{3 \tau}\right\}^{-3}$ & 6251 & $\mathrm{erg} \mathrm{cm}^{-3}$ \\
\hline radiative energy & $E_{\mathrm{rad}}=n_{0}^{2} \Psi_{0} T_{0}^{1 / 4}\left[1+\frac{1}{3 \tau}\right]^{-4}$ & 1.11 & erg $\mathrm{cm}^{-3} \mathrm{~s}^{-1}$ \\
\hline conductive energy & $E_{\text {cond }}=\frac{4 x_{0} T_{0}^{7 / 2}}{L^{2}}\left[1+\frac{1}{3 \tau}\right]^{-4}$ & 0.20 & $\operatorname{erg~cm} \mathrm{cm}^{-3} \mathrm{~s}^{-1}$ \\
\hline radiative timescale & $\tau_{\mathrm{rad}}=\tau_{\mathrm{rad}}(0)\left\{1+\frac{1}{3 \tau}\right\}$ & $\because$ & $s^{c l}$ \\
\hline conductive timescale & $\tau_{\text {cond }}=\tau_{\text {cond }}(0)\left\{1+\frac{1}{3 \tau}\right\}$ & $\tau / \mu$ & $s^{c l}$ \\
\hline & $\frac{\frac{1}{2} \rho v^{2}}{3 p / 2}=\left(\frac{13}{21}\right)^{2} \frac{\frac{1}{2} m_{H} n_{0} v_{0}^{2}}{3 n_{0} k T_{0}}\left(1+\frac{1}{3 t}\right\}^{-6 / 7}$ & 0.003 & \\
\hline density decrease & $\left(2 / \cup \int_{0}^{\infty} n v d t=\left(2 n_{0} v_{0} t\right) / L=n_{0}\right.$ & $2.610^{\prime \prime}$ & $\mathrm{cm}^{-3}$ \\
\hline
\end{tabular}

a) At $t=0$ the quast-static cooling phase starts. This corresponds $10 t=0$ in Fig. 2

b) $\left.v_{0}=L /(2 \tau)=142.2 \mathrm{~km} \mathrm{~s}^{-1} ; c\right) \tau=5618 \mathrm{~s} ., \mu=0.18$ 
Fig. 1. Ileight $/ I(\mathrm{~cm} r)$ of haring region as a function of the number $N$ of naring loops times the aspect ratio $\alpha=$ $(d / L)$ squared, normalized to a value of 0.1 as typically observed for Solar coronal loops. From a comparison with the ME observations $E M=9.510^{53} \mathrm{~cm}^{-3}$, $T=64 \mathrm{MK}$ and effective decay time $\tau_{e s s}$ $=5217 \mathrm{~s}$ were assumed. Also drawn are curves of constant $\mu^{\prime}: \mu^{\prime}=0.1,1,10$ (from Eqs. (1), (3)-(6): $\mu^{\prime}=r_{r} / r_{c} \simeq$ $0.00 H_{10} N c_{0.1}^{2}$ with $H_{10}$ in $\left.10^{10} \mathrm{~cm}\right)$.
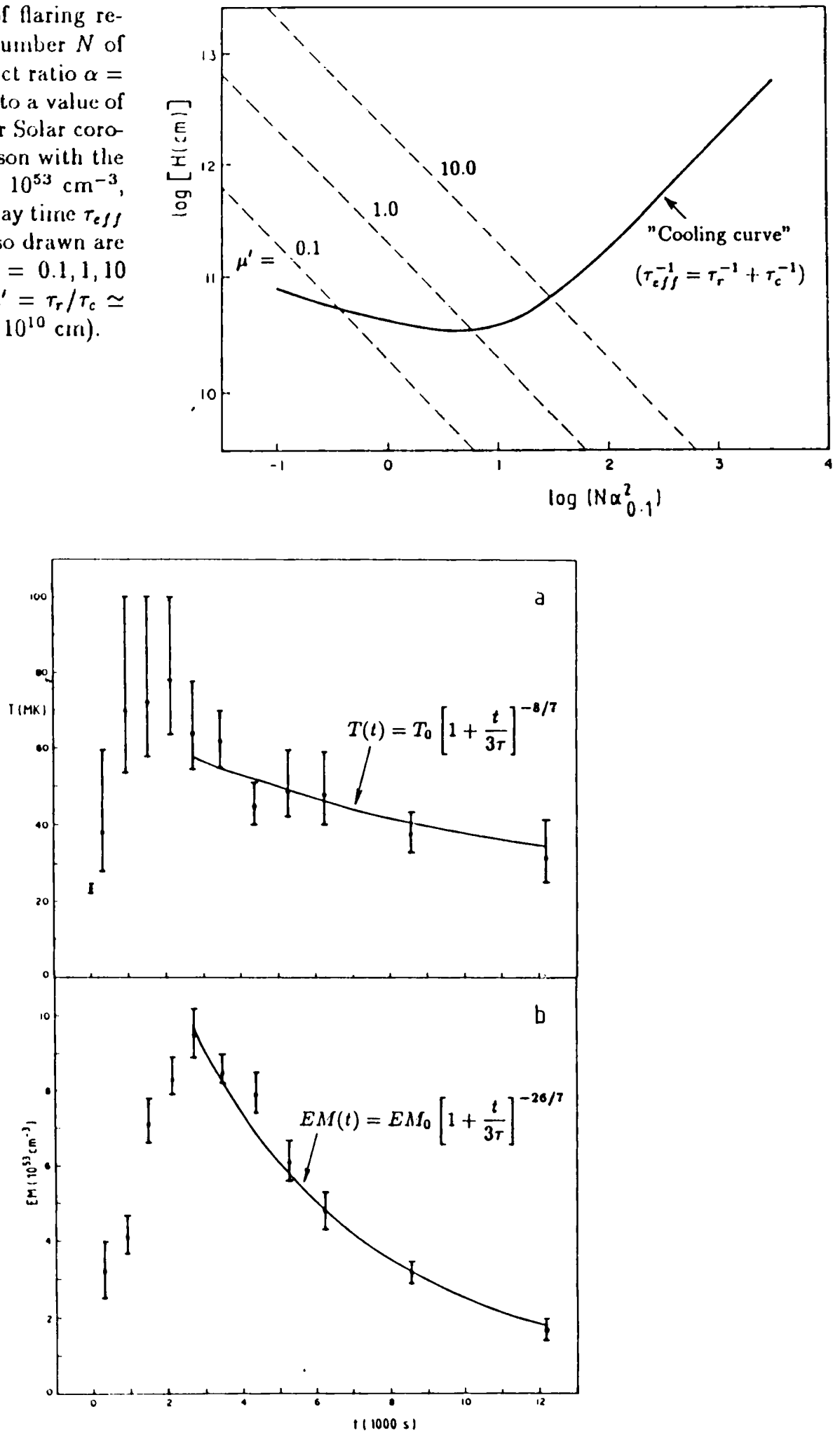

Fig. 2. Results of the fits to the observations for quasi-static cooling for temperature $T$ (panel a) and emission measure $E M$ (panel b). The best-fit parameters are $\varepsilon_{H}=0$ (no additional heating), $\tau=5618 \mathrm{~s}$, $T_{0}=57.6 \mathrm{MK}$ and $E M_{0}=9.810^{53} \mathrm{~cm}^{-3}$. Tine $t=0$ corresponds to $10: 20 \mathrm{UT}$ on 18 August 1983 . 